\title{
Is poor coordination of saccades in dyslexics a consequence of reading difficulties? A study case
}

\author{
Elham Ghassemi \\ IRIS Group, Centre d'Études de la \\ Sensori-Motricité (CESEM), \\ UMR 8194, CNRS, Université Paris \\ Descartes, Paris, France
}

\author{
Zoï Kapoula \\ IRIS Group, Centre d'Études de la \\ Sensori-Motricité (CESEM), \\ UMR 8194, CNRS, Service \\ d'ophtalmologie, Hôpital Européen \\ Georges Pompidou, Paris, France
}

\begin{abstract}
We hypothesize that the high quality of binocular coordination of saccades in reading is progressively learned during childhood, and this oculomotor learning is based on a synergy between saccades and vergence. In present work deficits in the binocular control of saccades in six dyslexic children (mean age was $11 \pm 2.48$ years) are studied for two tasks (text reading and $\mathrm{Xs}-\mathrm{C}$ scanning), and at two viewing distances $(40 \mathrm{~cm}$ and $100 \mathrm{~cm}$ ). Fixation durations resulting are longer in Xs-C scanning task than in text reading task. We postulate that while reading motor preparation processes are executed with less demand for attentional resources. Importantly all physiological parameters of the saccades were the same for the two conditions and in either distance. Namely disconjugacy of saccades and disconjugate post-saccadic drifts were high but similar for the two conditions. Time analysis applied on saccade amplitude disconjugacy, on disconjugate post-saccadic drift and on fixation duration showed no significant effect of repetition or time. we believe that the binocular coordination deficits in dyslexic children reflect some type of microdyspraxia due to reduced oculomotor learning, perhaps related to inefficiency of the magnocellular visual system and the cerebellar functions.
\end{abstract}

\section{Keywords: Binocular coordination, Developmental dyslexia, Cerebellum}

\section{Introduction}

Reading begins with vision. Vision is a prominently dynamic process because of saccadic and fixation eye movements. Saccades allow us to bring one word after another onto the fovea, where fine visual analysis takes place during the subsequent fixation period. Reading without saccades is abnormal. Bouma and De Voogd (1974) attempted artificial reading without saccades by presenting each word to fixating eyes. As they reported, although reading was still possible, it was slower; but comprehension was not assessed.

Research on eye movements in dyslexia follows two axes. The first deals with abnormalities in the regularities of eye movements during reading relative to non-dyslexic readers. For instance, shorter saccade sizes and longer fixation durations for dyslexics have often been reported
(Rayner, 1998; De Luca et al., 1999), as well as more regressive saccades (Pavlidis, 1981). Olson et al. (1991) reported that compared with normal readers such differences do not exist when dyslexics read pseudowords or during a non-lexical string processing task, suggesting that dyslexics' oculomotor abnormalities are caused by their reading difficulties rather than vice versa.

A second axis of research focuses on the oculomotor neurophysiology per se and uses simple tasks to stimulate eye movements, generally saccades. For instance, the latency of the saccades has been widely studied with accent on the occurrence of express types of latency (short latencies of 80-120 ms) that could be attributed to deficits in fixation control (Biscaldi et al., 1994). A few rather qualitative studies have described fixation instability, including poor vergence and deficits in binocular control (Stein et al., 1987). 
Reading on a bi-dimensional surface such as text or screen requires perfectly equally sized saccades of the two eyes. Such equality is important, so that the two eyes foveate the same letter and single fused binocular vision is obtained. But such requirement is not quite natural: in real life when we explore the real free space, most frequently we make saccades that are unequal for the two eyes, because the saccades also integrate vergence command. So how can we learn to make equal-sized saccades as they are required for reading? First it is believed that Hering's law of equal innervation of the two eyes is valid, although not perfect. The two eyes could receive the same saccade command, and are expected to make the same saccade amplitude. However there might be peripheral asymmetries in terms of the extra-ocular muscles or in terms of delays in the innervation circuit of each eye; such asymmetries will end up with a difference in the amplitude between the two eyes, even though the saccade command is the same for the two eyes. We hypothesize that the high quality of binocular coordination of saccades in reading is progressively learned during childhood, and this motor learning (neuroplasticity) is based on a synergy between saccades and vergence. According to this hypothesis the central nervous system has to learn to program both a conjugate but imperfect saccade command and a small vergence command. This small vergence that occurs during the saccade has the advantage in increasing the saccade size in one eye and decreasing it in the other eye, so that the final saccade becomes more equal for the two eyes (recall that during vergence each eye moves in opposite directions). This hypothetical motor learning occurs naturally during child development (Kapoula et al., 2008). Prior studies from the team (see for example, Yang and Kapoula, 2003) have shown that the binocular coordination during and after the saccades achieves adult levels at the age of about 10-12 years. For the younger children (4.5-6 years old) quality is poor, particularly at near distance. In contrast at far distance, the most dramatic improvement occurs at the age of about 7-8 years. Such distance dependency is in favor of the hypothesis mentioned above, according to which the binocular coordination is achieved via motor learning of saccade-vergence interaction. It has been shown that the deficits in the binocular coordination of saccades in dyslexic children are persisting beyond the age of 11-12 years old. Indeed the saccade disconjugacy (i.e., difference in saccade size between the two eyes) was found to be increased compared with age-matched nondyslexics in a single word reading task (Bucci et al., 2008), and this observation also held for free explorations of paintings (Kapoula et al., 2009). Moreover the saccade disconjugacy is typically followed by a disconjugate drift during the subsequent fixation which passively reduces the disconjugacy due to saccade. In dyslexic children the disconjugate drift during the fixation does not reduce the disconjugacy resulting from the saccade (Bucci et al., 2008). This saccade disconjugacy and disconjugate drifts during fixations are not correlated. This is another abnormality of the binocular control system in dyslexia.

We attribute this inefficiency in binocular coordination of saccades and the presence of disconjugate drift during fixations in dyslexic children to reduced saccade-vergence neuroplasticity. We consider this as a type of micro-dyspraxia that could be due to sensorimotor inefficiency. Specifically we hypothesize that the sensory magnocellular system - which is supposed to detect transient binocular disparities - is probably less efficient and does not provide appropriate error to the mechanism associated with oculomotor learning. Additionally the cerebellum which is one of the major parts of neuroplasticity is perhaps less efficient in dyslexia, and this could be the origin of reduced binocular coordination of saccades in dyslexics (see Kapoula et al., 2008).

Now let's return to the real text reading. Reading a text implies a sequence of saccades from left to right. Do deficits in the binocular control of saccades accumulate over successive saccades, or do they disappear because the sequence of saccades from left to right is over-trained and almost automatic? To answer this question, Jainta and Kapoula (2011) have examined saccades and fixations while reading a French text on a PC screen. Two groups of dyslexic (mean age was $11.7 \pm 2$ years) and nondyslexic (mean age was $12.7 \pm 1$ years) children participated in the study. The text reading task was performed at two viewing distances of $40 \mathrm{~cm}$ and 100 $\mathrm{cm}$. The authors reported more regressions for dyslexic children than non-dyslexics and longer fixation durations and larger saccade amplitudes in dyslexics than nondyslexics, presumably related to reading difficulties. They also reported larger saccade disconjugacy, as well as larger and more variable disconjugate post-saccadic drift for dyslexics than non-dyslexics. Jainta and Kapoula (2011) concluded that the deficits in the binocular coordination of saccades and disconjugate drift during fixations are intrinsic features of reduced neuroplasticity, and are not the consequence of reading difficulties. Kirkby et al. (2011) examined three participant groups of adults typically developing children (with a mean age of 9.89 years) and children with dyslexia (with a mean age of 10.41 years). They confirmed the study of Jainta and Kapoula, as they also found less precise binocular coordination and a significant increase in fixation disparity in dyslexic readers. However for dyslexic children, the binocular coordination of saccades 
depended on the task: the magnitude of fixation disparity resulting was greater than that found when they were scanning simple dot stimuli. The authors concluded that deficits in the binocular coordination result from reading difficulties, and are not primary physiological deficits. Earlier studies from the same group (see Kirkby et al., 2008; Blythe et al., 2006; Bucci et al., 2012) also reported abnormal patterns of eye movements in dyslexia attributing them to immaturity of saccade-vergence interaction, as introduced by Kapoula et al., (2008).

The objective of the present study is to reexamine this question in a small group of dyslexic children who are tested both during a text reading and a control condition task. This task involves scanning strings of Xs among which a C letter target is embedded. Moreover text reading and $\mathrm{Xs}-\mathrm{C}$ scanning task are tested at near and far viewing distances. It should be noted that the Xs-C scanning task involves only isolated letter recognition, while text reading task involves many other higher language processing (word processing, syntax, semantics, etc.).

\section{Methods}

\section{Ethics statement}

The investigation adhered to the principles of the Declaration of Helsinki, and was approved by a local internal ethics committee for human experimentation (CPP II de France II; No 07035; Hospital Necker, Paris). Informed oral consent was obtained from each child and his or her parents after clarification of the process of the experiment.

\section{Participants}

Six dyslexic children (mean age was $11 \pm 2.48$ years, 4 boys) officially classified as dyslexic by specialized schools or medical centers were tested. The classification evaluated their dyslexia condition with an extensive examination, including neurological/psychological and phonological capabilities made in the year of the present study (in 2011). For each child, the speed of reading, the text comprehension, and the capacity for reading words/pseudowords has been evaluated by using the L2MA battery (Chevrie-Muller et al., 1997). This is the standard test developed by the applied psychology center of Paris, and commonly used in France. It includes phonological fluency tests, a visual naming task, assessing the passive lexical stock, irregular words reading and spelling tasks. Generally the ability to use phonetic skills to decode words is evaluated by using the pseudoword reading test within the L2MA battery. Inclusion criteria were: (1) scores of reading abilities directly leading to a classification as dyslexic, i.e., scores in the L2MA test beyond two standard deviations; (2) a normal mean intelligence quotient stated in the written report, and (3) no neurological symptoms or ophthalmological pathologies.

All children had normal or corrected-to-normal vision. Binocular vision was assessed the day of eye movement measurements as stereo-thresholds based on disparity detection via the TNO random dot test (Netherlands Organization of Applied Scientific Research Test of stereoacuity); all individual scores were normal (60 seconds of arc or better).

\section{Tasks and stimuli}

\section{'L'alouette' text reading task}

The child was seated comfortably in an adjusted chair, and her/his head was stabilized with a chin rest. She/he viewed binocularly the TFT screen on which, the "L'alouette"' text (in French) appeared in black letters on white background. The "L'alouette", is commonly used in France for the evaluation of reading capacity in dyslexia. It contains non-frequent words and the order of the words is unusual in French; the reader cannot use anticipation (Lefavrais, 1967). The text was written in Times New Roman (in font size 12) and each letter was about 0.3 degrees of angular size. Six text panels (heights $\times$ width) of $8 \times 10$ degrees were presented in sequence on the screen, covering the integral of "L'alouette" text. Each panel contained 8 lines of text double spaced. To ensure that subjects actually read the text, they were asked to briefly comment on it. In common with adults (Vernet and Kapoula, 2009), the children complained about the strangeness of the text and cited few words or parts of the context.

The children had to read the " $L$ 'alouette" at near (40 $\mathrm{cm})$ and far $(100 \mathrm{~cm})$ viewing distances. The text size was rescaled according to the distance and the sequence of the text presentations.

\section{Xs and Cletter target scanning task}

In this task each letter of the "L'alouette" text was replaced by the " $\mathrm{X}$ " character, except the central character that was replaced by a " $C$ ". The child was asked to scan the lines of $\mathrm{X}$ characters, and to fixate each $\mathrm{C}$ letter target. The task implied a sequence of saccades 
from left to right similar to that activated during text reading task.

As the $\mathrm{C}$ target was in the middle or to the left of each $\mathrm{Xs}$ chain, it required a fixation in the middle, similarly to what happens during real reading; it is known that the eyes are fixating slightly left of the word's center (see Vitu et al., 1990).

The Xs and C were also written in Times New Roman (in font size 12) and each letter was about 0.3 degrees of angular size. This task was also done at near $(40 \mathrm{~cm})$ and far $(100 \mathrm{~cm})$ viewing distances.

\section{Apparatus and calibration}

Oculomotor data for the left and right eye were measured dynamically $(200 \mathrm{~Hz})$, using a head-fixed (i.e., head mounted) infrared video eye tracker (Chronos Vision, Berlin); a chin rest was used to stabilize the children's head. The Chronos eye tracking system records digital image sequences and evaluates offline eye position variations with a reported resolution of less than 0.1 degrees.

Before each reading block, a standard saccadic paradigm was used to elicit visually guided saccades: a target (two segments of $0.9 \times 0.7$ degrees aligned with offsets of 0.1 degrees vertically and 0.7 degrees horizontally) jumped between five positions on the screen (at the center and at \pm 8 degrees horizontally and vertically). The subject was asked to follow accurately the center of the target (at the offset space). Stable fixation periods between saccades were used to extract the calibration factors, separately for each eye. Viewing during calibrations was monocular (i.e., one eye was occluded with a patch), and each saccade target was presented four times for each eye.

\section{Data analysis}

Calibration and analysis methods were similar to those used in prior studies (Vernet and Kapoula, 2009; Jainta and Kapoula, 2011). Briefly a linear function was used to convert eye position signals into degrees. From the separate signals of the two eyes we calculated the conjugate eye movements [(left eye + right eye $) / 2$; i.e., the version signal] and the disconjugate eye movements [left eye - right eye; i.e., the vergence signal]. The onset or offset of horizontal saccades were defined as the time, when the eye velocity of the conjugate signal exceeded or dropped below, respectively $10 \%$ of the maximum velocity.
We extracted several parameters from the eye movement signals. For each detected saccade, we calculated its amplitude as difference in the version signal between the ending of the saccade (E) and its beginning (B), (markers E and B, in figure 1.a). More importantly we extracted the change in the vergence between the saccade on- and offsets (see Bucci et al., 2008; Vernet and Kapoula, 2009); this change in the vergence signal between the markers $\mathrm{E}$ and $\mathrm{B}$, in figure $1 . \mathrm{b}$ gives $\mathrm{a}$ measure of saccade disconjugacy.

Further knowing the saccade on- and offsets, we defined fixation periods between saccades as real fixations as long as they were longer than $80 \mathrm{~ms}$ and shorter than $500 \mathrm{~ms}$. The end of a fixation period was marked by an $\mathrm{F}$ and this end was defined as $10 \mathrm{~ms}$ before the next saccade started (see figure 1.a). For the fixation period, we also calculated the disconjugate post-saccadic drift (Bucci et al., 2008), i.e., the changes in vergence between the beginning of the fixation period (E) and the final position (F) before the next saccade (markers [E-F], in figure 1.b). Thus short or long fixation durations $(<80$ $\mathrm{ms}$ and $>500 \mathrm{~ms}$ ), saccade amplitudes smaller than 0.5 degrees, as well as saccades or fixations contaminated by blinks were not included in the analysis; rejection rates on these criteria were similar for all tasks (30\% to $40 \%)$.

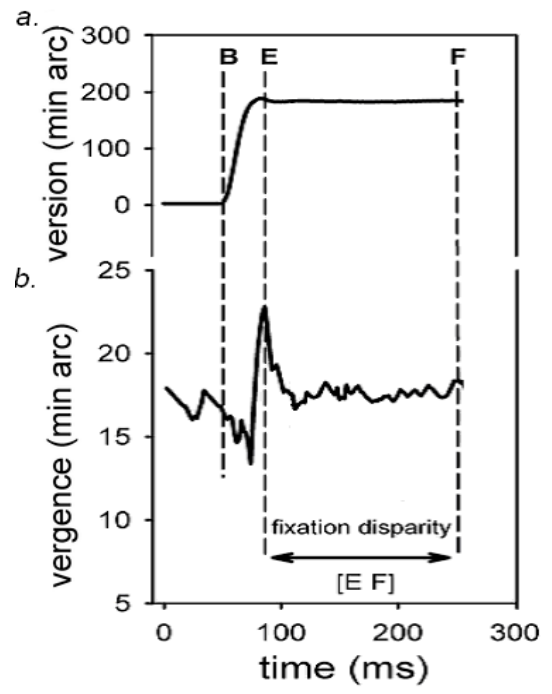

Figure 1. Example of saccade while reading. In a. the version signal ((right eye + left eye) /2; min arc); the beginning of the saccade was marked by $a B$ and its end by an E. Further the end of the fixation period was marked by an $F$ and it was defined as $10 \mathrm{~ms}$, before the beginning of the next saccade. All markers from the version signal were transferred into the vergence signal. In b. the vergence signal (left eye - right eye; min arc) is shown. 
Additionally in order to check fixation durations, we calculated them for each fixation period [E-F]. We included only forward (i.e., rightward) saccades into our analysis.

\section{Statistical analysis}

\section{Comparison between text reading and $\mathrm{Xs}$-C letter target scanning tasks}

Results will be presented for the following parameters: saccade amplitude, mean saccade velocity (amplitude/duration), fixation duration, saccade disconjugacy and disconjugate post-saccadic drift. For each of these parameters, we applied the Friedman, nonparametric test (four conditions: text reading at near, text reading at far, Xs-C scanning at near, Xs-C scanning at far). The Wilcoxon test was done only when the Friedman test was significant. We also used values of Cohen's $d$ (Cohen, 1988) to compare text reading and Xs$\mathrm{C}$ scanning tasks at two distances.

\section{Time analysis}

In order to account for eventual effects of repetition, we used a linear mixed-effects model [lmer from package lme4 (Pinheiro and Bates, 2000; Venables and Smith, 2001) in R (http://www.r-project.org)]. Generally linear mixed-effects models are based on maximum likelihood methods, and commonly used in many areas (Baayen et al., 2008).

The statistical package $\mathrm{R}$ provides reliable algorithms for mixed effect parameter estimations, as well as tools for their evaluation (West et al., 2007): the $p$-values and confidence intervals were estimated by using posterior distributions for the model parameters obtained by Markov Chain Monte Carlo sampling, including typically a sample size of 10000 (see Baayen et al., 2008). In our study separate estimations were made for each of the following variables: saccade disconjugacy, disconjugate post-saccadic drift and fixation duration. We defined the saccade or fixation number for each individual subject as the repetition, fixed effect, while subjects were treated as random effect (i.e., individual differences in the intercept were taken into account). We estimated the coefficient with its standard error $(S E)$, the $t$-value and the $p$-value for each effect of repetition.

\section{Results}

\section{Fixation Duration}

Table 1 shows the individual and group means of fixation duration for each of the four conditions. The Friedman test showed a significant effect $(F(3,6)=9.40$, $p<0.02)$. Fixation duration was shorter in the text reading than in the $\mathrm{Xs}-\mathrm{C}$ scanning task. Comparisons done with the Wilcoxon test showed that fixation duration was significantly higher for the Xs-C scanning than for the text reading at both distances, $(p<0.02)$.

The measure of the effect size - Cohen's $d$ value (comparing text reading and Xs-C scanning tasks) - was 0.19 at near distance and 0.38 at far distance, indicating that the effect was trivial-small at near distance and small-medium at far distance.

Table 1. Individual and group mean and standard deviation (SD) of fixation duration (in ms) for each of the four conditions

\begin{tabular}{ccc}
\hline Subjects & $\begin{array}{c}\mathrm{X} \pm \mathrm{SD}, \mathrm{n} \text { for } \\
\text { Text }-40 \mathrm{~cm} .\end{array}$ & $\begin{array}{c}\mathrm{X} \pm \mathrm{SD}, \mathrm{n} \text { for } \\
\text { Text }-100 \mathrm{~cm}\end{array}$ \\
\hline S1 & $211 \pm(86) 77$ & $218 \pm(94) 91$ \\
S2 & $227 \pm(85) 168$ & $236 \pm(79) 174$ \\
S3 & $222 \pm(95) 133$ & $236 \pm(101) 106$ \\
S4 & $219 \pm(89) 204$ & $229 \pm(96) 219$ \\
S5 & $219 \pm(74) 140$ & $207 \pm(74) 62$ \\
S6 & $256 \pm(109) 65$ & $234 \pm(95) 22$ \\
\hline Mean & $226 \pm(90)$ & $227 \pm(90)$
\end{tabular}

(a) Text reading task at near and far distances

\begin{tabular}{ccc}
\hline Subjects & $\begin{array}{c}\mathrm{X} \pm \mathrm{SD}, \mathrm{n} \text { for } \\
\mathrm{X}-40 \mathrm{~cm}\end{array}$ & $\begin{array}{c}\mathrm{X} \pm \mathrm{SD}, \mathrm{n} \text { for } \\
\mathrm{X}-100 \mathrm{~cm}\end{array}$ \\
\hline S1 & $239 \pm(102) 63$ & $224 \pm(93) 88$ \\
S2 & $247 \pm(92) 230$ & $284 \pm(102) 124$ \\
S3 & $251 \pm(93) 220$ & $267 \pm(111) 129$ \\
S4 & $264 \pm(106) 198$ & $281 \pm(120) 115$ \\
S5 & $212 \pm(111) 24$ & $217 \pm(104) 59$ \\
S6 & $252 \pm(110) 104$ & $308 \pm(105) 20$ \\
\hline Mean & $244 \pm(102)$ & $264 \pm(106)$ \\
\hline
\end{tabular}

(b) Xs-C scanning task at near and far distances

\section{Saccade Amplitude}

Table 2 shows the results of saccade amplitude for each of the four conditions. Saccade amplitudes were similar in both the text reading and the Xs-C scanning tasks. The Friedman test showed no significant effect, $(F$ $(3,6)=0.98, p<0.81)$. 
The measure of the effect size - Cohen's $d$ value was 0.41 at near distance and 0.50 at far distance, indicating that the effect was medium for both distances.

Table 2. Individual and group mean and standard deviation (SD) of saccade amplitude, for each of the four conditions

\begin{tabular}{ccc}
\hline Subjects & $\begin{array}{c}\mathrm{X} \pm \mathrm{SD}, \mathrm{n} \text { for } \\
\text { Text }-40 \mathrm{~cm}\end{array}$ & $\begin{array}{c}\mathrm{X} \pm \mathrm{SD}, \mathrm{n} \text { for } \\
\text { Text }-100 \mathrm{~cm}\end{array}$ \\
\hline S1 & $2.16 \pm(1.30)$ & $2.07 \pm(1.18)$ \\
S2 & $2.63 \pm(1.16)$ & $2.45 \pm(1.13)$ \\
S3 & $1.89 \pm(0.95)$ & $2.00 \pm(1.13)$ \\
S4 & $2.59 \pm(0.97)$ & $2.40 \pm(0.97)$ \\
S5 & $2.35 \pm(1.22)$ & $2.49 \pm(1.32)$ \\
S6 & $1.56 \pm(1.02)$ & $2.07 \pm(1.05)$ \\
\hline Mean & $2.20 \pm(1.10)$ & $2.25 \pm(1.13)$ \\
\hline
\end{tabular}

(a) Text reading task at near and far distances; $n$ for each subject is the same as in Table 1.a

\begin{tabular}{ccc}
\hline Subjects & $\begin{array}{c}\mathrm{X} \pm \mathrm{SD}, \mathrm{n} \text { for } \\
\mathrm{X}-40 \mathrm{~cm}\end{array}$ & $\begin{array}{c}\mathrm{X} \pm \mathrm{SD}, \mathrm{n} \text { for } \\
\mathrm{X}-100 \mathrm{~cm}\end{array}$ \\
\hline S1 & $1.82 \pm(1.06)$ & $2.00 \pm(1.11)$ \\
S2 & $1.91 \pm(1.11)$ & $2.30 \pm(0.85)$ \\
S3 & $2.16 \pm(1.11)$ & $2.03 \pm(1.01)$ \\
S4 & $2.50 \pm(1.13)$ & $2.28 \pm(1.11)$ \\
S5 & $1.85 \pm(0.55)$ & $1.74 \pm(1.01)$ \\
S6 & $1.76 \pm(1.05)$ & $1.73 \pm(0.94)$ \\
\hline Mean & $2 \pm(1)$ & $2.01 \pm(1.01)$ \\
\hline
\end{tabular}

(b) Xs-C scanning task at near and far distances; $n$ for each subject is the same as in Table 1.b

\section{Mean Saccade Velocity}

Table 3 shows the results of mean saccade velocity for each of the four conditions. The Friedman test showed no significant effect $(F(3,6)=5.01, p<0.17)$.

The measure of the effect size was -0.04 at near distance and -0.46 at far distance, indicating that there was no really effect at near distance, but a medium effect at far distance.

Table 3. Individual and group mean and standard deviation (SD) of mean saccade velocity for each of the four conditions

\begin{tabular}{ccc}
\hline Subjects & $\begin{array}{c}\mathrm{X} \pm \mathrm{SD}, \mathrm{n} \text { for } \\
\text { Text }-40 \mathrm{~cm}\end{array}$ & $\begin{array}{c}\mathrm{X} \pm \mathrm{SD}, \mathrm{n} \text { for } \\
\text { Text }-100 \mathrm{~cm}\end{array}$ \\
\hline S1 & $-46 \pm(22)$ & $-53 \pm(22)$ \\
S2 & $-74 \pm(30)$ & $-73 \pm(29)$ \\
S3 & $-54 \pm(23)$ & $-54 \pm(23)$ \\
S4 & $-77 \pm(27)$ & $-71 \pm(27)$ \\
S5 & $-63 \pm(31)$ & $-79 \pm(59)$
\end{tabular}

\begin{tabular}{cll} 
S6 & $-40 \pm(22)$ & $-54 \pm(19)$ \\
\hline Mean & $-59 \pm(26)$ & $-64 \pm(30)$ \\
\hline
\end{tabular}

(a) Text reading task at near and far distances; $n$ for each subject is the same as in Table 1.a

\begin{tabular}{ccc}
\hline Subjects & $\begin{array}{c}\mathrm{X} \pm \mathrm{SD}, \mathrm{n} \text { for } \\
\mathrm{X}-40 \mathrm{~cm}\end{array}$ & $\begin{array}{c}\mathrm{X} \pm \mathrm{SD}, \mathrm{n} \text { for } \\
\mathrm{X}-100 \mathrm{~cm}\end{array}$ \\
\hline S1 & $-52 \pm(42)$ & $-53 \pm(26)$ \\
S2 & $-57 \pm(30)$ & $-38 \pm(13)$ \\
S3 & $-60 \pm(25)$ & $-53 \pm(20)$ \\
S4 & $-76 \pm(32)$ & $-70 \pm(32)$ \\
S5 & $-55 \pm(17)$ & $-57 \pm(26)$ \\
S6 & $-48 \pm(30)$ & $-39 \pm(17)$ \\
\hline Mean & $-58 \pm(29)$ & $-52 \pm(22)$ \\
\hline
\end{tabular}

(b) Xs-C scanning task at near and far distances; $n$ for each subject is the same as in Table 1.b

\section{Saccade Disconjugacy}

Table 4 shows the saccade disconjugacy for each subject in the four conditions tested. The saccade disconjugacy was substantial, on average $>0.2$ degrees, but this was the case for all conditions. The Friedman test showed no significant effect $(F(3,6)=1.06, p<0.78)$.

The measure of the effect size at near distance was 0.16 and at far distance was 0.14 , indicating that the effect was nearly trivial for the two distances.

Table 4. Individual and group mean and standard deviation (SD) of saccade amplitude disconjugacy for each of the four conditions

\begin{tabular}{ccc}
\hline Subjects & $\begin{array}{c}\mathrm{X} \pm \mathrm{SD}, \mathrm{n} \text { for } \\
\text { Text }-40 \mathrm{~cm}\end{array}$ & $\begin{array}{c}\mathrm{X} \pm \mathrm{SD}, \mathrm{n} \text { for } \\
\text { Text }-100 \mathrm{~cm}\end{array}$ \\
\hline S1 & $0.29 \pm(0.26)$ & $0.21 \pm(0.20)$ \\
S2 & $0.15 \pm(0.11)$ & $0.14 \pm(0.10)$ \\
S3 & $0.13 \pm(0.10)$ & $0.52 \pm(0.32)$ \\
S4 & $0.29 \pm(0.23)$ & $0.21 \pm(0.14)$ \\
S5 & $0.37 \pm(0.31)$ & $0.43 \pm(0.35)$ \\
S6 & $0.15 \pm(0.15)$ & $0.32 \pm(0.13)$ \\
\hline Mean & $0.23 \pm(0.19)$ & $0.31 \pm(0.21)$ \\
\hline
\end{tabular}

(a) Text reading task at near and far distances; $n$ for each subject is the same as in Table 1.a

\begin{tabular}{ccc}
\hline Subjects & $\begin{array}{c}\mathrm{X} \pm \mathrm{SD}, \mathrm{n} \text { for } \\
\mathrm{X}-40 \mathrm{~cm}\end{array}$ & $\begin{array}{c}\mathrm{X} \pm \mathrm{SD}, \mathrm{n} \text { for } \\
\mathrm{X}-100 \mathrm{~cm}\end{array}$ \\
\hline S1 & $0.35 \pm(0.33) 63$ & $0.24 \pm(0.23) 81$ \\
S2 & $0.12 \pm(0.11) 230$ & $0.23 \pm(0.16) 124$ \\
S3 & $0.17 \pm(0.11) 220$ & $0.48 \pm(0.31) 129$ \\
S4 & $0.24 \pm(0.20) 198$ & $0.18 \pm(0.13) 115$ \\
S5 & $0.37 \pm(0.30) 24$ & $0.38 \pm(0.34) 59$
\end{tabular}




\begin{tabular}{ccc} 
S6 6 & $0.13 \pm(0.11) 104$ & $0.14 \pm(0.14) 20$ \\
\hline Mean & $0.20 \pm(0.19)$ & $0.28 \pm(0.22)$ \\
\hline
\end{tabular}

(b) $X s$-C scanning task at near and far distances; $n$ for each subject is the same as in Table 1.b

\section{Disconjugate post-saccadic drift}

Table 5 shows the results for disconjugate postsaccadic drift. Again such drtift was high $>0.2$ degrees, but this was the case for all conditions. The Friedman test showed no significant effect $(F(3,6)=0.98, p<0.8)$.

The measure of the effect size at near distance was 0.04 and at far distance was 0 , indicating that there was no really effect.

Table 5. Individual and group means and standard deviation (SD) of disconjugate post-saccadic drift for each of the four conditions

\begin{tabular}{ccc}
\hline Subjects & $\begin{array}{c}\mathrm{X} \pm \mathrm{SD}, \mathrm{n} \text { for } \\
\text { Text }-40 \mathrm{~cm}\end{array}$ & $\begin{array}{c}\mathrm{X} \pm \mathrm{SD}, \mathrm{n} \text { for } \\
\text { Text }-100 \mathrm{~cm}\end{array}$ \\
\hline S1 & $0.53 \pm(0.58)$ & $0.33 \pm(0.34)$ \\
S2 & $0.23 \pm(0.15)$ & $0.19 \pm(0.19)$ \\
S3 & $0.13 \pm(0.11)$ & $0.24 \pm(0.37)$ \\
S4 & $0.29 \pm(0.23)$ & $0.21 \pm(0.17)$ \\
S5 & $0.37 \pm(0.31)$ & $0.56 \pm(0.49)$ \\
S6 & $0.15 \pm(0.15)$ & $0.39 \pm(0.13)$ \\
\hline Mean & $0.28 \pm(0.26)$ & $0.32 \pm(0.28)$ \\
\hline
\end{tabular}

(a) Text reading task at near and far distances; $n$ for each subject is the same as in Table 1.a

\begin{tabular}{ccc}
\hline Subjects & $\begin{array}{c}\mathrm{X} \pm \mathrm{SD}, \mathrm{n} \text { for } \\
\mathrm{X}-40 \mathrm{~cm}\end{array}$ & $\begin{array}{c}\mathrm{X} \pm \mathrm{SD}, \mathrm{n} \text { for } \\
\mathrm{X}-100 \mathrm{~cm}\end{array}$ \\
\hline S1 & $0.35 \pm(0.34)$ & $0.43 \pm(0.45)$ \\
S2 & $0.20 \pm(0.17)$ & $0.30 \pm(0.25)$ \\
S3 & $0.13 \pm(0.11)$ & $0.17 \pm(0.24)$ \\
S4 & $0.19 \pm(0.16)$ & $0.18 \pm(0.12)$ \\
S5 & $0.54 \pm(0.44)$ & $0.70 \pm(0.62)$ \\
S6 & $0.23 \pm(0.31)$ & $0.15 \pm(0.14)$ \\
\hline Mean & $0.27 \pm(0.26)$ & $0.32 \pm(0.30)$ \\
\hline
\end{tabular}

(b) Xs-C scanning task at near and far distances; $n$ for each subject is the same as in Table 1.b

\section{Time analysis}

The rational was to test whether the parameters studied (the saccade amplitude disconjugacy, the disconjugate post-saccadic drift and the fixation duration) increase over time due to fatigue. Howeve for all these parameters there was no statistical significant effect of repetition or time ( $p$-values for all regression measures were $>0.1$ and for all parameters). This was the case for both text reading and $\mathrm{Xs}-\mathrm{C}$ scanning tasks and at both viewing distances (see Tables 7-9 in Annex).

\section{Discussion}

This study aimed to evaluate whether increased saccade amplitude disconjugacy and disconjugate postsaccadic drift in dyslexic children are due to reading difficulties, or if they are due to intrinsic physiological deficits. To answer this question, a groupe of six dyslexic children was tested in two conditions while reading a text ("L'alouette") or scanning the Xs-C chain. The Xs-C scanning task involved similar sequence of saccades as the text reading task. These two conditions were done both at near and at far distances. An important point is the nature of our Xs-C scanning task. As mentioned, this task requires isolated letter recognition and no word processing. Therefore this is far different from text reading task that requires more language processing. Our observations are limited to this type of control. The main results are the following. First, there is a cognitive effect on fixation duration. Fixation durations are longer in the $\mathrm{Xs-C}$ scanning task than in the text reading task. In contrast, all physiological parameters of the saccades were the same for the two conditions. Namely the results showed similar saccade amplitude in the two conditions and in either distance $(40 \mathrm{~cm}$ or $100 \mathrm{~cm})$. Saccade disconjugacy and disconjugate post-saccadic drift which are important in dyslexics were similar in two conditions (text reading or Xs-C scanning) and in either distance. Finally time analysis applied on saccade amplitude disconjugacy, on disconjugate post-saccadic drift and on fixation duration showed no significant effect of repetition or time. Due to reduced number of subjects, the effects are not always strong (see Cohen's $d$ values). Therefore the present study are rather preliminary and require further studies. Next we will discuss these results, starting first with fixation duration and subsequently with the results of saccade disconjugacy.

\section{Fixation duration}

Fixation duration is longer for the $\mathrm{Xs}-\mathrm{C}$ scanning task than for the text reading task. Fixation duration includes the time needed to prepare the next saccade. The fact that fixation duration is longer in the Xs- $\mathrm{C}$ condition indicates that this process of next saccade preparation is of lower time cost when it occurs in parallel with reading. In other words, when language processing is involved as in a text reading, the next point of fixation is almost automatically 
done. It is important to note that the saccade amplitudes were the same in the two conditions; the text reading and the Xs-C scanning. This result indicates some type of crossmodal interaction between processes on saccade programing and fixation control on the one hand, and on the other hand, language reading process. While reading motor preparation processes are executed more automatically; i.e., with less demand for attentional resources.

Now we will switch over to the binocular coordination aspects, i.e., the saccade disconjugacy and the disconjugate post-saccadic drift which are the main focus of this study.

\section{Binocular coordination aspects}

First one should question whether disconjugacy values reported here in dyslexics are abnormal. In this study no controls were included. However a comparison cand be made with the data published by Jainta \& Kapoula (2011), using the same setup for a group of control children with comparable age (mean age: 12.7 \pm 1 years; 4 girls, 3 boys). Table 6 summarises the means and the standard deviation of disconjugacy values for controls and for dyslexics reported here. It is clear that all values are higher for dyslexics than for controls, confirming the deficits in the binocular coordination in dyslexia. Next we will discuss these aspects.

Table 6. Means and standard deviation (SD) of saccade amplitude disconjugacy and disconjugate post-saccadic drift for text reading task at two distances

\begin{tabular}{ccc}
\hline & \multicolumn{2}{c}{ Near $(40 \mathrm{~cm})$} \\
\cline { 2 - 3 } & $\begin{array}{c}\text { Saccade disconjgacy } \\
(\mathrm{deg})\end{array}$ & $\begin{array}{c}\text { Disconjugate post- } \\
\text { saccadic drift (deg) }\end{array}$ \\
\hline $\begin{array}{c}\text { Controls } \\
(\mathrm{n}=7)\end{array}$ & $0.12 \pm(0.05)$ & $0.11 \pm(0.02)$ \\
\hline $\begin{array}{c}\text { Dyslexics } \\
(\mathrm{n}=6)\end{array}$ & $0.23 \pm(0.19)$ & $0.28 \pm(0.26)$ \\
\hline \multicolumn{3}{c}{ Far $(100 \mathrm{~cm})$} \\
\cline { 2 - 3 } & $\begin{array}{c}\text { Saccade disconjugacy } \\
(\text { deg })\end{array}$ & $\begin{array}{c}\text { Disconjugate post- } \\
\text { saccadic drift (deg) }\end{array}$ \\
\hline $\begin{array}{c}\text { Controls } \\
(\mathrm{n}=7)\end{array}$ & $0.14 \pm(0.06)$ & $0.11 \pm(0.05)$ \\
\hline $\begin{array}{c}\text { Dyslexics } \\
(\mathrm{n}=6)\end{array}$ & $0.31 \pm(0.21)$ & $0.32 \pm(0.28)$ \\
\hline
\end{tabular}

As mentioned, the saccade amplitudes were the same in the two conditions and at two viewing distances. Moreover there was no time repetition effect on neither of these parameters. All these results show clearly the deficits in binocular coordination of saccades, and that maintaining the binocular coordination during fixation by avoiding disconjugate post-saccadic drifts are intrinsic, as these problems exist independently from reading. The results that we report here are clearly in opposition with the study of Kirkby et al. (2011). Yet our control Xs-C task is different from that used by Kirkby et al. (2011) sequences of dot stimuli. If the binocular coordination deficits are a consequence of reading difficultries, as suggested by Kirkby et al. (2011), then our study shows that this is the case even only elementary process are involved such as individual letter recognition without word and text processing. Alternatively our control study shows that disconjugacy is always present independetly from text reading. Further comparative studies with many different controls in the same children would be of interest. Our interpretation of the present data is that the binocular coordination deficits in dyslexic children reflect some type of micro-dyspraxia due to reduced oculomotor neuroplasticity. This reduced neuroplasticity could be related to reduced synergy between neural mechanisms subserving saccade-vergence interaction. Perhaps the deficits relate to the magnocellular visual system which is supposed to detect transient binocular disparity, and transfer the disparity error signal(s) to the cerebellum for oculomotor adjustments. This interpretation is in line with the magnocellular theory of developmental dyslexia by John Stein (2001). In addition to this inefficiency of the magnocellular system, we belive that the cerebellum which is a major side of oculomotor plasticity could also be inefficient particularly in relation to time aspects.

The fact that these deficits exist for either viewing distance is also indicative of the severity of the problem, and indicates that efficient binocular oculomotor adjustments are not possible in dyslexia no mater what the reading distance is. It should be noted that in another population, for instance, in children with vertigo without vestibular pathologies, have shown the binocular coordination deficits to be limited to the near distance only (Bucci et al., 2009). It seems that in dyslexics, the deficits are more generalized, and are not specific to the depth. Also in prior studies in healthy children (Kapoula et al., 2008), we have shown that maturation of the binocular coordination with age depends on the depth, i.e., it is achieved for far distance than for near distance (7-8 years vs. 11-12 years of age). This is why we expected dyslexics would show the binocular coordination deficits more at near distance. The results show that even for far distance, the binocular coordination deficits persist in dyslexia.

To summarize, our results provide further strong evidence for the existence of the binocular oculomotor 
control deficits independently from reading. These observations are in line with our prior studies (Kapoula et al., 2008; Bucci et al., 2008) that show that dyslexics have the deficits of binocular coordination and of disconjugate drifts during fixations even when they are doing rudimentary saccade tasks, like saccading to a single target. The binocular control deficits reflect microdyspraxia which is perhaps related to inefficiency of the magnocellular and of the cerebellar functions.

\section{Acknowledgments}

The authors wish to thank Stephanie Jainta for her contributions on the experiments and also Chrystal Gaertner for her statistical support on Friedman and Wilcoxon tests.

\section{References}

Baayen, R. H., Davidson, D. J., \& Bates, D. M. (2008). Mixed-effects modeling with crossed random effects for subjects and items. Journal of Memory and Language, 59, 390-412.

Biscaldi, M., Fischer, B., \& Aiple, F. (1994). Saccadic eye movements of dyslexic and normal reading children. Perception, 23(1), 45-64.

Blythe, H. I., Liversedge, S. P., Joseph, H. S. S. L., White, S. J., Findlay, J.M. \& Rayner, K. (2006). The binocular coordination of eye movements during reading in children and adults. Vision Research, 46(22), 3898-3908.

Bouma, H., \& De Voogd, A. H. (1974). On the control of eye saccades in reading. Vision Research, 14(4), 273284.

Bucci, M. P., Brémond-Gignac, D., \& Kapoula, Z. (2008). Poor binocular coordination of saccades in dyslexic children. Graefes Archive for Clinical and Experimental Ophthalmology, 246(3), 417-428.

Bucci, M. P., Lê, T. T., Wiener-Vacher, S., BrémondGignac, D., Bouet, A., \& Kapoula, Z. (2009). Poor postural stability in children with vertigo and vergence abnormalities. Investigative Ophthalmology \& Visual Science, 50(10), 4678-4684.

Bucci, M. P., Nassibi, N., Gerard, C.-L., Gerard, BuiQuoc, E., \& Seassau, M. (2012). Immaturity of the Oculomotor Saccade and Vergence Interaction in Dyslexic Children: Evidence from a Reading and Visual Search Study. PLoS ONE, 7(3): e33458.
Chevrie-Muller, C., Simon, A. M., \& Fournier, S. (1997). Batterie Langage oral écrit, Mémoire, Attention (L2MA). Paris: Éditions du Centre de Psychologie Appliquée.

Cohen, J. (1988). Statistical Power Analysis for the Behavioral Sciences ( $2^{\text {nd }}$ ed.). Hillsdale, New Jersey: Lawrence Erlbaum Associates, Publishers.

De Luca, M., Di Pace, E., Judica, A., Spinell, D., \& Zoccolotti, P. (1999). Eye movement patterns in linguistic and non-linguistic tasks in developmental surface dyslexia. Neuropsychologia, 37(12):1407-1420.

Jainta, S., \& Kapoula, Z. (2011). Dyslexic Children Are Confronted with Unstable Binocular Fixation while Reading. PLoS ONE, 6(4):e18694.

Kapoula, Z., Ganem, R., Poncet, S., Gintautas, D., \& Eggert, T. (2009). Free exploration of painting uncovers particularly loose yoking of saccades in dyslexics. Dyslexia, 15(3), 243-259.

Kapoula, Z., Vernet, M., Yang, Q., \& Bucci, M. P. (2008). Binocular coordination of saccades: development, aging and cerebral substrate. Journal of Eye Movement Research, 2(3):3, 1-20.

Kirkby, J. A., Webster, L. A. D., Blythe, H. I., \& Liversedge, S. P. (2008). Binocular coordination during reading and non-reading tasks. Psychological Bulletin, 134(5), 742-763.

Kirkby, J. A., Blythe, H. I., Drieghe, D., \& Liversedge, S. P. (2011). Reading Text Increases Binocular Disparity in Dyslexic Children. PLoS ONE, 6(11): e27105.

Lefavrais, P. (1967). Test de l'Alouette. Paris: Éditions du Centre de Psychologie Appliquée ( $2^{\text {ème }}$ éd.).

Olson, R. K., Conners, F. C., \& Rack, J. P. (1991). Eye movements in dyslexic and normal readers. In Stein, J. F. (Ed.), Vision and Visual Dysfunction (pp. 243250). London: Macmillan.

Pavlidis, G. T. (1981). Do eye movements hold the key to dyslexia? Neuropsychologia, 19(1), 57-64.

Pierrot-Deseilligny, C., Muri, R. M., Rivaud-Pechoux, S., Gaymard, B., \& Ploner, C. J. (2002). Cortical control of spatial memory in humans: the visuooculomotor model. Annals of Neurology, 52(1), 10-19.

Pinheiro, J. C., Bates, D. M. (2000). Mixed-Effects Models in S and S-Plus (Statistics and Computing). New York: Springer-Verlag. 
R-Development-Core-Team, (2008). R: A Language and Environment for Statistical Computing. Retrieved from http://www.r-project.org

Rayner, K. (1998). Eye movements in reading and information processing: 20 years of research. Psychological Bulletin, 124(3), 372-422.

Stein, J. F., Riddell, P. M., \& Fowler, M. S. (1987). Fine binocular control in dyslexic children. Eye, $1(\mathrm{Pt} 3)$, 433-438.

Stein, J. F. (2001). The magnocellular theory of developmental dyslexia. Dyslexia, 7(1), 12-36.

Venables, W. N., \& Smith, D. M. (2001). An Introduction to R. Notes on R: A Programming Environment for Data Analysis and Graphics. Version 1.4.0 (200112-19. Retrieved from http://nitro.biosci.arizona.edu/r/RManual/R-intro.pdf

Vernet, M., \& Kapoula, Z. (2009). Binocular motor coordination during saccades and fixations while reading: a magnitude and time analysis. Journal of Vision, $9(7): 2,1-13$.

Vitu, F., O'Regan, J. K., \& Mittau, M. (1990). Optimal landing position in reading isolated words and continuous text. Perception \& Psychophysics, 47(6), 583-600.

West, B. T., Welch, K. B., \& Gallechki, A. T. (2007). Linear Mixed Models: A Practical Guide Using Statistical Software. Boca Raton, Florida: Chapman and Hall/CRC Press.

Yang, Q., \& Kapoula, Z. (2003). Binocular coordination of saccades at far and at near in children and in adults. Journal of Vision, 3(8):3, 554-561.

\section{Annex}

Table 7. Saccade disconjugacy

\begin{tabular}{ccc}
\cline { 2 - 3 } & \multicolumn{2}{c}{$\mathrm{X}-40 \mathrm{~cm}$} \\
\cline { 2 - 3 } & (Intercept) & Trials \\
\hline Estimate & 0.0055 & 0.0001 \\
SE & 0.0808 & 0.0013 \\
t-Value & 0.062 & 0.600 \\
p-Value & 0.9506 & 0.5530 \\
lower limit & -0.1402 & -0.0002 \\
upper limit & 0.1470 & 0.0004 \\
\hline & \multicolumn{2}{c}{$\mathrm{X}-100 \mathrm{~cm}$} \\
\cline { 2 - 3 } & (Intercept) & Trials \\
\hline
\end{tabular}

\begin{tabular}{ccc}
\hline Estimate & -0.0982 & -0.0006 \\
SE & 0.0838 & 0.0004 \\
t-Value & -1.172 & -1.446 \\
p-Value & 0.2084 & 0.1328 \\
lower limit & -0.2621 & -0.0014 \\
upper limit & 0.0739 & 0.0002 \\
\hline & \multicolumn{2}{c}{ Text $-40 \mathrm{~cm}$} \\
\cline { 2 - 3 } & $($ Intercept $)$ & Trials \\
\hline Estimate & 0.0091 & 0.0002 \\
SE & 0.0739 & 0.0002 \\
t-Value & 0.123 & 0.746 \\
p-Value & 0.8776 & 0.4584 \\
lower limit & -0.1609 & -0.0003 \\
upper limit & 0.1806 & 0.0006 \\
\hline & \multicolumn{2}{c}{ Text $-100 \mathrm{~cm}$} \\
\cline { 2 - 3 } & $($ Intercept $)$ & Trials \\
\hline Estimate & -0.1583 & 0.0002 \\
SE & 0.0757 & 0.0002 \\
t-Value & -2.093 & 0.832 \\
p-Value & 0.0524 & 0.4222 \\
lower limit & -0.3065 & -0.0003 \\
upper limit & 0.0079 & 0.0006 \\
\hline & &
\end{tabular}

Table 8. Disconjugate post-saccadic drift

\begin{tabular}{|c|c|c|}
\hline & \multicolumn{2}{|c|}{$\mathrm{X}-40 \mathrm{~cm}$} \\
\hline & (Intercept) & Trials \\
\hline Estimate & 0.0525 & -0.0001 \\
\hline $\mathrm{SE}$ & 0.0683 & 0.0002 \\
\hline t-Value & 0.768 & -0.761 \\
\hline p-Value & 0.4116 & 0.4606 \\
\hline lower limit & -0.0941 & 0.2064 \\
\hline \multirow[t]{3}{*}{ upper limit } & -0.0005 & 0.0002 \\
\hline & \multicolumn{2}{|c|}{$\mathrm{X}-100 \mathrm{~cm}$} \\
\hline & (Intercept) & Trials \\
\hline Estimate & 0.1381 & 0.0008 \\
\hline $\mathrm{SE}$ & 0.0597 & 0.0006 \\
\hline t-Value & 2.314 & 1.341 \\
\hline p-Value & 0.0756 & 0.1818 \\
\hline lower limit & -0.0145 & -0.0004 \\
\hline \multirow[t]{3}{*}{ upper limit } & 0.2994 & 0.0018 \\
\hline & \multicolumn{2}{|c|}{ Text $-40 \mathrm{~cm}$} \\
\hline & (Intercept) & Trials \\
\hline Estimate & 0.1269 & -0.0001 \\
\hline SE & 0.0392 & 0.0003 \\
\hline t-Value & 3.234 & -0.455 \\
\hline p-Value & 0.0210 & 0.6542 \\
\hline lower limit & 0.0307 & -0.0007 \\
\hline \multirow[t]{3}{*}{ upper limit } & 0.2324 & 0.0004 \\
\hline & \multicolumn{2}{|c|}{ Text $-100 \mathrm{~cm}$} \\
\hline & (Intercept) & Trials \\
\hline Estimate & 0.1192 & 0.0001 \\
\hline SE & 0.0493 & 0.0003 \\
\hline
\end{tabular}


Journal of Eye Movement Research $6(1): 5,1-11$

\begin{tabular}{ccc}
$\mathrm{t}-$ Value & 2.418 & 0.458 \\
$\mathrm{p}-$ Value & 0.0706 & 0.6424 \\
lower limit & -0.0166 & -0.0004 \\
upper limit & 0.2425 & 0.0007 \\
\hline
\end{tabular}

Table 9. Fixation duration

\begin{tabular}{|c|c|c|}
\hline & \multicolumn{2}{|c|}{$\mathrm{X}-40 \mathrm{~cm}$} \\
\hline & (Intercept) & Trials \\
\hline Estimate & 252 & -0.0220 \\
\hline $\mathrm{SE}$ & 6.65 & 0.0556 \\
\hline t-Value & 37.92 & -0.39 \\
\hline p-Value & 0.0001 & 0.6042 \\
\hline lower limit & 235.02 & 268.11 \\
\hline \multirow[t]{3}{*}{ upper limit } & -0.1462 & 0.0785 \\
\hline & \multicolumn{2}{|c|}{$\mathrm{X}-100 \mathrm{~cm}$} \\
\hline & (Intercept) & Trials \\
\hline Estimate & 250.98 & 0.2314 \\
\hline SE & 15.0987 & 0.1432 \\
\hline t-Value & 16.623 & 1.616 \\
\hline p-Value & 0.0001 & 0.0992 \\
\hline lower limit & 213.1164 & -0.0397 \\
\hline \multirow[t]{3}{*}{ upper limit } & 287.4154 & 0.5154 \\
\hline & \multicolumn{2}{|c|}{ Text $-40 \mathrm{~cm}$} \\
\hline & (Intercept) & Trials \\
\hline Estimate & 222.7001 & 0.0246 \\
\hline SE & 6.9899 & 0.0670 \\
\hline t-Value & 31.86 & 0.37 \\
\hline p-Value & 0.0001 & 0.7216 \\
\hline lower limit & 205.2472 & -0.1107 \\
\hline \multirow[t]{3}{*}{ upper limit } & 239.8137 & 0.1555 \\
\hline & \multicolumn{2}{|c|}{ Text $-100 \mathrm{~cm}$} \\
\hline & (Intercept) & Trials \\
\hline Estimate & 230.45 & -0.0205 \\
\hline SE & 6.7789 & 0.0680 \\
\hline t-Value & 34.0 & -0.3 \\
\hline p-Value & 0.0001 & 0.7606 \\
\hline lower limit & 214.2247 & -0.1627 \\
\hline upper limit & 248.6617 & 0.1106 \\
\hline
\end{tabular}

Ghassemi and Kapoula (2013)

Is poor coordination of saccades in dyslexics a consequence of reading difficulties? A study case 